Meta

Journal des traducteurs

Translators' Journal

\title{
Ariettes retrouvées, contes recréés
}

Quelques aspects de la création chez Césaire dans ses rapports

avec l'oralité

\section{Lilian Pestre de Almeida}

Volume 31, numéro 3, septembre 1986

Prismes de traductions littéraires

Facets of Literary Translation

URI : https://id.erudit.org/iderudit/001875ar

DOI : https://doi.org/10.7202/001875ar

Aller au sommaire du numéro

Éditeur(s)

Les Presses de l'Université de Montréal

ISSN

0026-0452 (imprimé)

1492-1421 (numérique)

Découvrir la revue

Citer cet article

Pestre de Almeida, L. (1986). Ariettes retrouvées, contes recréés : quelques aspects de la création chez Césaire dans ses rapports avec l'oralité. Meta, 31(3), 272-290. https://doi.org/10.7202/001875ar d'utilisation que vous pouvez consulter en ligne.

https://apropos.erudit.org/fr/usagers/politique-dutilisation/ 


\section{ARIETTES RETROUVÉES, CONTES RECRÉÉS QUELQUES ASPECTS DE LA CRÉATION CHEZ CÉSAIRE DANS SES RAPPORTS AVEC L'ORALITÉ}

LILIAN PESTRE DE ALMEIDA

UNIVERSIDADE FEDERAL FLUMINENSE, RIO DE JANEIRO, BRÉSIL

\section{En hommage à Luis da Câmara Cascudo}

Les rapports de Césaire avec l'oralité (ou l'oraliture) sont loin d'être résolus ou même cernés dans toute leur complexité. Devant l'instabilité du texte césairien (en particulier du Cahier qui ne cesse de bouger pendant vingt ans), nous avons émis jadis l'hypothèse d'un lien, plus profond qu'on ne le croit, de la poésie de Césaire avec la parole orale ${ }^{1}$. Refusant de figer son texte ne varietur, le poète sans qu'il l'affirme publiquement ou même qu'il en ait conscience, se présente en quelque sorte comme l'héritier du griot ou du conteur populaire. D'autre part, il n'est plus nécessaire, nous semble-t-il, d'avancer des exemples de la présence de l'oraliture dans la poésie et le théâtre de Césaire. Nous en avons indiqué ailleurs quelques exemples qui vont de la simple citation de comptines à la paraphrase occulte de petits contes ${ }^{2}$. Mais cette présence, discrète dans les premiers recueils de poèmes et "naturelle » dans son théâtre, car il fallait faire parler des personnages "populaires " (Hugonin dans le Roi Christophe ou le joueur de sanza dans Une saison), devient plus évidente et palpable dans ses derniers poèmes.

Il est évident que ce bref essai n'a rien à voir avec ce que Thomas A. Hale appelle littérature orale chez Césaire : pour le critique américain, il s'agit d'étudier le discours du poète en tant que conférencier, candidat ou tribun ${ }^{3}$. Or, sans qu'il y ait là le goût du paradoxe, le discours analytique, idéologique ou politique de Césaire, bien que prononcé " oralement » devant un auditoire, relève de toute évidence de la littérature écrite. Oralité pour nous se rattache à une production collective orale, c'est-à-dire à des formes traditionnelles et populaires comme le conte, l'énigme, la devinette, le proverbe, etc. Et nous y incluons les chants pour saluer les orishas, pontos comme on le dit en portugais, et les orikis (invocations rituelles).

Cette étude peut paraître une gageure ou une témérité (elle l'est en grande partie) pour une personne non créolophone comme c'est notre cas. Elle naît cependant d'une certaine sensibilité à l'oral que partagent, croyons-nous, les différentes cultures américaines. En effet, les rapports entre l'oralité et l'écriture sont au centre même des littératures dans les Amériques, qu'elles s'écrivent en français, en espagnol ou en portugais. Un phénomène comme celui de l'œuvre de Guimaräes Rosa au Brésil n'est possible que dans un contexte d'oralité encore très vivace. Nous pourrions multiplier les titres d'œuvres brésiliennes où un extraordinaire travail d'écriture est traversé par la prégnance de l'oral : un exemple récent en est le long récit d'Autran Dourado, Novelário de Donga Novais $^{4}$, construit tout entier à partir de courts proverbes, texte où le langage d'un écri- 
vain contemporain "érudit " se crée à partir du langage collectif, le je individuel se pliant au nous collectif.

Précisons bien nos objectifs et nos limites. Il ne s'agit pas ici d'explorer exhaustivement, dans le travail de l'écriture césairienne, les traces de créolisme (présence ou absence du créole derrière le français, lapsus révélateurs, inscription et déconstruction de la proverbialité créole, etc.). Il ne s'agit pas non plus de situer le poème césairien dans un contexte de diglossie littéraire, telle qu'elle apparaît dans des récits comme ceux de Jacques Roumain, Simone Schwartz-Bart ou Édouard Glissant analysés, dans les deux premiers cas, par des créolophones comme Maximilien Laroche ${ }^{5}$ ou Jean Bernabé6. Notre projet est tout autre. Il s'agit de voir dans quelle mesure les poèmes de Césaire, en particulier ceux de son dernier recueil moi, laminaire, même pour une étrangère, relèvent de l'oralité à travers l'emploi de certaines formes ou personnages populaires, et par là se lient à une poétique américaine. Car l'impression la plus immédiate et la plus forte que nous a laissée moi, laminaire... tient au fait que, dans de très courts poèmes, Césaire s'américanise. Et c'est cela que notre texte voudra expliciter.

Lilyane Kesteloot, la première, a dégagé le rapport entre un poème du recueil Ferrements intitulé "Beau sang giclé " et le conte populaire d'Yé qui, grimpant sur un palmier, tue l'oiseau magique qui ressuscitera réclamant le compte de ses plumes dispersées. Nous citons le poème de Ferrements suivi du commentaire de Kesteloot :

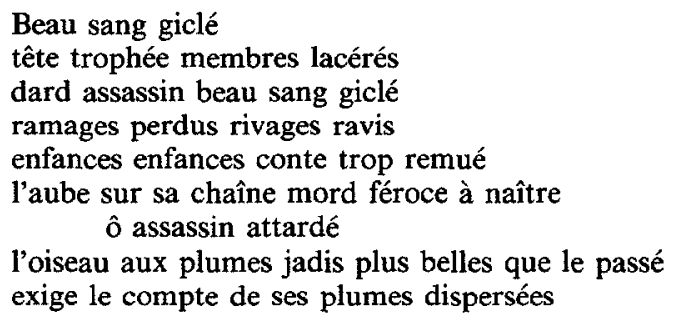

Commentaire.

Sur la même lancée que le précédent poème, Césaire reprend ici le conte de l'oiseau enchanté que Yé, le paysan, avait tué par mégarde et qui, après avoir été mangé, ressuscita et obligea l'homme, sa femme et ses enfants à lui rendre jusqu'à sa dernière plume.

Césaire en fait l'allégorie du peuple nègre, mutilé, martyrisé comme un gibier (tête trophée, membres lacérés, beau sang giclê), privé de ses langues et de sa culture (ramages perdus) et exilé par la Traite (rivages ravis).

Mais cette histoire de son peuple a trop bercé ses enfances, comme un triste négrospiritual. Aujourd'hui, l'aube, c'est-à-dire la vie nouvelle, se prépare avec impatience (mord sur sa chaine) et avec violence.

$O$ assassin attardé : avertissement au maître qui a assassiné sa race, et se trouve toujours sur les lieux du crime.

L'oiseau ... exige le compte de ses plumes dispersées : les nègres se révoltent et réclament tout ce qu'on leur a pris d'humanité.

(In Aimé Césaire. L'homme et l'œuvre, pp. 58-59.)

Le commentaire de L. Kesteloot, que nous citons intégralement, constitue en fait une explicitation politique à plat du poème qui n'a pas grand chose en commun, malgré ce qu'en affirme Kesteloot, avec le poème précédent. La forme elliptique et ramassée de "Beau sang giclé » contraste avec l'ampleur rhétorique du poème dédié à Lafcadio Hearn. Et le critique qui ne s'occupe pas des champs sémantiques, ne se pose même pas les problèmes de la disparition totale de la triade humaine du conte antillais (Yé, sa 
femme, et une " rafale d'enfants ") et de l'apparition d'un " assassin attardé ». Kesteloot ne fait pas non plus allusion au jeu (inversé) de la dévoration : dans le conte, c'est Yé et sa famille qui dévorent l'oiseau; dans le poème, la gueule agressive appartient à l'aube (qui "sur sa chaîne mord féroce à naître ") et à l'oiseau qui s'exprime par la parole. Césaire qui donne la piste de l'oraliture à son lecteur par le vers " enfances enfances conte trop remué ", abandonne la structure du conte.

Ce poème néanmoins est important parce qu'il permet de voir qu'en plus d'un intérêt pour la littérature antillaise traditionnelle (voir l'article écrit dès 1942 en collaboration avec René Ménil sur la littérature orale ainsi que de nombreuses publications sur le sujet dans la revue Tropiques), Césaire incorpore à ses poèmes des thèmes de contes.

Cependant c'est dans le tout dernier recueil de Césaire, où de très courts poèmes abondent, que nous trouvons des pièces qui ne peuvent être lues sans l'intertextualité de l'oralité. Nous en détachons quelques-unes : le no 24 ("ça, le creux»), le $n^{\circ} 38$ (" inventaire de cayes ") auxquels il faudrait ajouter le $n^{\circ} 10$ ("chanson de l'hippocampe »), le $\mathrm{n}^{\circ}$ 42 ("internonce »), le $n^{\circ} 23$ ("mot-macumba") et de petits poèmes qui font partie du tombeau de Wifredo Lam, le peintre cubain, "conversation avec Mantonica Wilson", " connaître, dit-il ", " que l'on présente son cœur au soleil ".

Nous adressant aussi à un public brésilien et croyant qu'une des meilleures voies de pénétration dans un texte réside dans une proposition de traduction dans la langue maternelle, nous faisons suivre chacun des courts poèmes césairiens d'une version en portugais ${ }^{7}$, justifiant, le cas échéant, certaines solutions.

Nous commençons par le poème, à première vue, le plus obscur de l'ensemble, "ça, le creux";

ça, le creux

ça ne se meuble pas

c'est le creux

ça ne s'arrache pas

ce n'est pas une fleur

ça s'effilocherait plutôt

étoupe pour étouffer les cris

(s'avachissant ferme)

ça se traverse

tunnel

- pas forcément à toute vitesse -

ça se gravit aussi en montagne

glu

le plus souvent ça se rampe

Nous proposons comme traduction en portugais :

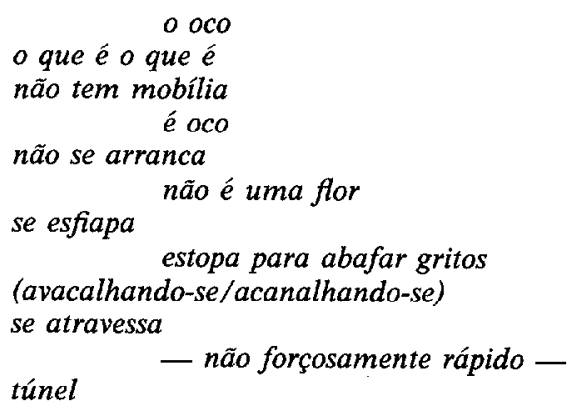




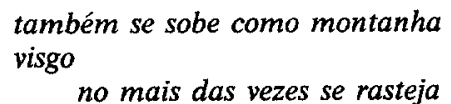

Dans la traduction en portugais nous faisons précéder le texte de la formule brésilienne de la devinette : «o que é o que é », car il nous semble qu'il y a, dans le poème césairien, une ellipse évidente. Le conteur/poète cache la formule traditionnelle antillaise qui ouvrait la voie au jeu des devinettes : cric, crac. Que la réponse soit l'île natale (ou plutôt l'Histoire subie dans l'île natale), le lecteur ne sera pas loin de la réponse. Et trouver une réponse n'est pas, comme nous le verrons, anodin : une réponse (ou la réponse) permet de vivre.

La devinette naît de l'idée d'énigme, elle évoque pour les Occidentaux l'image angoissante d'OEdipe devant le Sphynx : une énigme lourde de contrainte qu'il faut déchiffrer sous peine de mort. Les dieux grecs quand ils daignaient parler, le faisaient de manière obscure qui exigeait des interprétations : déchiffrer l'oracle c'était résoudre des devinettes. L'oracle de Delphes dit qu'on donne l'armée à l'homme aux trois yeux : Ternemos donne le commandement des troupes à un borgne à cheval; le salut de la Grèce était sur les tranchées en bois : les Grecs sont vainqueurs à Salamine, car les bateaux étaient des tranchées en bois. Les exemples en sont multiples. Les formules sont brèves, dans la plupart des cas susceptibles de métrification pour en faciliter l'assimilation mnémonique. Elles répercutent d'ailleurs d'une langue à l'autre :

$\begin{array}{ll}\text { Cai da torre } & \text { Cae de una torre } \\ \text { não se lasca; } & \text { y no se lastima; } \\ \text { cai na água } & \text { entra en el rio } \\ \text { se despedaça. (o papel) } & \text { y se vuelve harina. (el papel) }\end{array}$

Dans la tradition nagô, si importante dans les Amériques, analysée par des chercheurs tels que Roger Bastide, Pierre Verger, Juana Elbein dos Santos et Lydia Cabrera, il y a bon nombre d'énigmes qu'il faut résoudre. L'une de ces énigmes appartient à l'histoire d'Odu Ogbe Ogunda, reproduite par Pierre Verger et reprise par Juana Elbein dos Santos ${ }^{8}$. Le conte est très long mais l'essentiel réside dans une énigme qu'Orunmila doit étre capable de résoudre pour garantir son existence et celle des êtres humains. Il doit deviner le sens de la phrase : "Elles disent, lancer ; Orunmila dit, attraper " et ceci 7 fois. Orunmila répond qu'elles vont lancer un œuf 7 fois et qu'il devra l'attraper dans de la bourre de coton. Orunmila est pardonné et les enfants des êtres humains également. Juena Elbein dos Santos commente :

Les naissances peuvent continuer. Pour que la vie des êtres continue, Orunmila doit connaître l'énigme de la fécondation, le rapport de l'œuf avec la bourre de coton. L'œuf, élément-signe féminin, doit être "attrapé " par la bourre de coton, élément-signe masculin. L'énigme résolue, "attraper un cuf dans de la bourre de coton " signifie féconder Iyá-mi, établir le rapport harmonieux féminin-masculin qui rend possible la continuité de l'existence. (Os nagô e a morte, Vozes, 1976, p. 112.)

Nous avons choisi l'énigme de l'œuf, car elle nous permettra plus tard de lire un autre poème de Césaire ("que l'on présente son cœur au soleil ").

La prédilection des Nègres pour les devinettes est abondamment notée par les voyageurs en Afrique. Le Père Bouche cité par Rocha Pombo (História do Brasil, II, 485) témoigne :

Dans les réunions, les nègres s'amusent avec des jeux d'esprit. Proposer et déchiffrer des énigmes est un divertissement fort prisé parmi les nègres, même chez les enfants. Ils y em- 
ploient des heures et des heures. Ils en ont de fort intéressantes par l'exactitude des caractéristiques.

Consultant adivinhação dans le Dicionário de Folclore Brasileiro de Câmara Cascudo, pourtant fort riche, nous n'y avons pas trouvé d'indication de la fonction même de la devinette, jeu enfantin qui, sous la trivialité, est une forme, croyons-nous, de distraire l'angoisse face à la mort. C'est un jeu qui, même dans le monde profane, permet d'éluder la mort. Deux exemples pris à des récits francophones, volontairement très distants l'un de l'autre, nous permettront de mieux comprendre la fonction (occulte) du jeu des devinettes. Dans Gouverneurs de la rosée 9 , le soir de la veillée funèbre de Manuel, les femmes prient ou pleurent, les hommes jouent aux cartes (p. 194) tandis que le Simidor Antoine joue aux devinettes avec les jeunes (p. 196). Avant que le prêtre savane et le hougan Dorméus ne viennent pour recommander le cadavre du héros, Jacques Roumain fait le tour des activités populaires en Haïti pendant une veillée funèbre : les cartes, les cantiques, les devinettes. De façon similaire, dans un autre roman, $\mathrm{Thé} \mathrm{da}^{10}{ }^{10}$, de $\mathrm{S}$. Corinna Bille, dont l'action se passe dans un petit village du Valais, la narratrice raconte comment, enfant, elle a failli mourir pour avoir bu l'eau d'un étang maléfique : sa longue convalescence se passe à jouer aux devinettes avec la femme de son frère aîné (Théoda, chap. IX intitulé "Les devinettes", pp. 74-75). Les passages qui décrivent des réalités paysannes, articulés soit avec les énigmes posées à OEdipe ou à Orunmila, soit avec les pièges des oracles grecs, permettent de dévoiler la fonction cachée des devinettes, jeu puéril souvent lié à la mort elle-même. La devinette devient alors un exercice initiatique, pauvre ruse des hommes devant la Mort qui les menace. Savoir la réponse permet de vivre et de vaincre la mort. Ces brèves suggestions qui viennent de deux textes contemporains fort éloignés (Haïti : Roumain ; le Valais : Corinna Bille) mais enracinés tous les deux dans le monde paysan, dans les rites du temps liturgique (vodoun et catholique) en contact étroit avec le cycle des saisons (pour Haïti : carême et hivernage; pour le Valais : automne, hiver, printemps, été) nous aident à mieux situer le petit texte mystérieux "ça, le creux".

Considérons donc l'atmosphère générale du texte qui nous intéresse : par la forme même de la devinette, le poème de Césaire se place d'emblée dans l'univers du sacré. Le lecteur/auditeur devra imaginer un être en danger, gravement malade, agonisant ou cadavre, et que pour le sauver, il devra trouver la réponse. Suivons les termes de l'énigme qui nous est proposée, " ça, le creux ". Le vide (" ça ne se meuble pas "), sa persistance (" ça ne s'arrache pas »), le discontinu et la déchéance du quotidien vécu ("ça s'effilocherait plutôt ", "s'avachissant ferme "), l'impossibilité du cri dans des bouches bâillonnées ( étoupe pour étouffer les cris »), le paysage en tant que simple lieu de passage et non pas d'enracinement (" ça se traverse - pas forcément à toute vitesse »), le long trou noir d'où on ne sort jamais et d'où l'on ne perçoit point la lumière (« tunnel "), la présence et la soif de la montagne ("ça se gravit aussi en montagne "), la viscosité et le gras de la mangrove ("glu le plus souvent ça se rampe ") tracent en traits elliptiques l'espace où vit l'Antillais. Dans l'énigme césairienne, les deux derniers verbes (gravir, ramper) renvoient à deux manières d'être et d'agir du héros césairien : celui qui s'élève et monte, celui qui s'arc-boute dans le gras de la boue : Christophe/Metellus.

Le texte du poème $n^{\circ} 38$ "inventaire de cayes» est plus simple :

inventaire de cayes

beaux

(à siffler sur la route)

beaux

Caraibos 


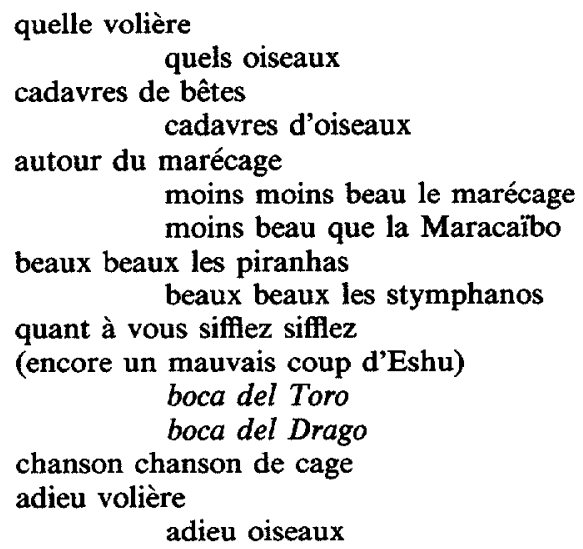

Nous en proposons la version en portugais :

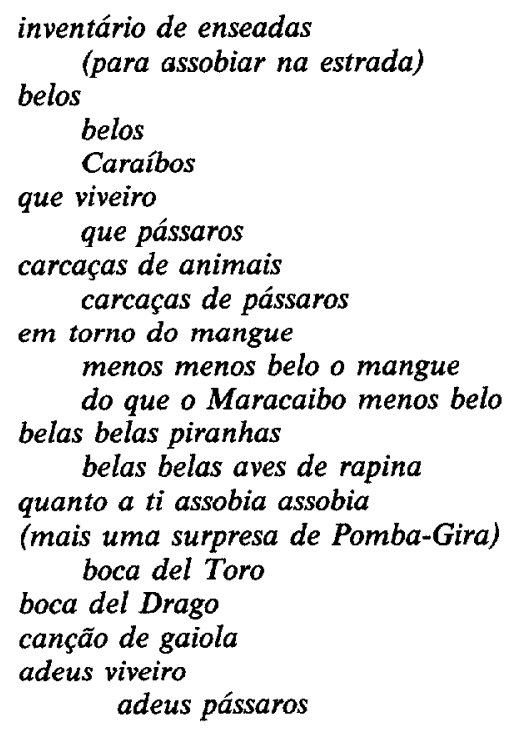

Une brève note sur la traduction. En portugais, le mot piranha étant féminin et l'adjectif bela devant s'accorder, les jeux sonores sur les $o$ ont été remplacés par des jeux sonores sur des $i$, voyelle d'ailleurs plus agressive que la voyelle $o$ finale (prononcée [u]) : piranha, rapina, ti, assobia, Pomba-Gira. La substitution d'Eshu par sa "femme" Pompa-Gira dans la macumba brésilienne renforce l'aire sémantique de l'agressivité et de la rapacité s'articulant avec les féminins piranhas, aves, rapina ${ }^{11}$.

Dans le poème $n^{0} 38$ tout suggère, dès la première lecture, une ariette où le narrateur-siffleur fait le tour, non pas de l'île natale, mais des Caraïbes. Comme en plaisantant, dans une chanson-comptine il joue sur les mots étrangers, la syntaxe redondante du créole, court-circuitant les signifiés grâce aux jeux des sonorités et des allusions. Le mot mystérieux stymphano est saisi à la fois à partir du Styx (Nom d'une fontaine d'Arcadie, fameuse par le froid extrême de ses eaux, qui étaient mortelles, disait-on, pour ceux qui en buvaient. Fleuve qui, selon la mythologie, coulait aux enfers; les dieux ju- 
raient par le Styx, et ce serment ne pouvait être violé) et du Stymphale (lac célèbre de la mythologie par les oiseaux monstrueux aux becs et aux serres d'airain vivant sur ses bords et qui se nourrissaient de chair humaine : Héraclès les fit sortir de leur retraite à l'aide des cymbales et les tua à coups de flèches). Dans cet "inventaire de cayes", le multilinguisme caribéen se fait jour à travers l'espagnol (Caraibos, Maracaibo, boca del Toro, boca del Drago), le portugais (piranhas) et la prégnance de la syntaxe créole. Cet inventaire qui est celui des serres, dents, becs agressifs et mutilants (des stymphanos et des piranhas), de la mort subie (anéantissement des Caraïbes, de l'oiseau magique mangé par Yé, de Colibri tué), aboutit à la grande gueule dévoratrice de la Bête non nommée (boca del Toro, boca del Drago ${ }^{12}$ ). Mais cet inventaire du malheur est inversé au moment même où il s'explicite grâce aux sifflements du marcheur sur sa route, Eshu "qui ouvre les chemins", "le maître des carrefours". Fidèle à son rôle de médiateur, Eshu est source de changement, de dialectisation :

Eshu! La pierre qu'il a lancée hier

c'est aujourd'hui qu'elle tue l'oiseau.

Du désordre il fait l'ordre, de l'ordre le désordre!

$\mathrm{Ah}$ ! Eshu est un mauvais plaisant.

Le passage, fort connu, de la pièce Une tempête (III, 3, p. 70) n'est que la citation, littérale, d'orikis d'Eshu.

La même silhouette d'Eshu apparaît, en filigrane, dans le poème $n^{\circ} \mathbf{4 2}$ du même recueil, "internonce". En voici le texte :

\section{Internonce}

il m'arrive de le perdre

des semaines

c'est ma créature mais rebelle

un petit mot couresse

un petit mot crabe-c'est-ma-faute

un petit mot pétale de feu

un petit mot pétrel plongeur

un petit mot saxifrage de tombeaux

petit mot qui m'atteste je te lance tiaulé

dans le temps et les confins

assistant à ton assaut sévère

spectral et saccadé

et de mon sang luciole parmi les lucioles

La traduction en portugais pourrait être la suivante :

\section{Internúncio}

acontece-me perdê-la

durante semanas

é minha criatura mas rebelde

uma palavra cobra d'água

uma palavra caranguejo

uma palavra pétala de fogo

uma palavra procelária-negra-do-norte

uma palavra saxifrágia de túmulos 


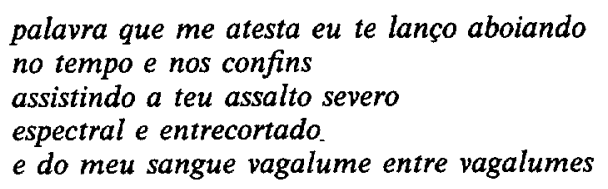

Le lecteur pourrait se demander où se trouve Eshu dans ce poème. Or, nous venons de faire allusion à un oriki d'Eshu recueilli par Pierre Verger et repris par Césaire dans Une tempête : le même geste de lancer une pierre contre les contraintes de l'espacetemps (" Eshu ! La pierre qu'il a lancée hier / c'est aujourd'hui qu'elle tue l'oiseau ") apparaît sous la forme allusive de lancer " un petit mot " dans le temps et les confins". D'ailleurs, dans le système nagô, Eshu est le principe même de la communication.

Dans le poème, le petit mot - "c'est ma créature mais rebelle " - atteste l'existence de celui qui le lance : il est à la fois serpent (" couresse "), oiseau vorace vivant sur la haute mer (" pétrel plongeur "), plante herbacée qui brise les pierres tombales (" saxifrage de tombeaux "), crabe, pétale et feu. Il est lancé " tiaulé », c'est-à-dire en chantant selon le Littré. Aboiando, dirions-nous en portugais.

Tiaulé : aboiando. C'est un des rares cas, nous semble-t-il, où les résonances de la version brésilienne sont infiniment plus larges que celles de l'original césairien au niveau du lecteur moyen (Français ou Brésilien). Tiauler, tiaulement restent des mots rares en français et la définition qu'en donne le Littré est fort pauvre tandis que les mots brésiliens aboio, aboiar ont toute la charge connotative qui vient non seulement d'une tradition littéraire (orale et écrite) importante dans notre culture mais encore du fait qu'ils correspondent à une réalité concrète, celle des vaqueiros du Nord-Est ou de l'intérieur de Minas Gerais. Le lecteur brésilien a dans sa mémoire émotive le ton de la mélopée du cavalier qui guide et dompte son troupeau de bœufs en chantant. Et l'aboio a ses règles et ses tabous. Comme l'indique Câmara Cascudo :

Dans le sertão du Brésil l'aboio est toujours solo, chant individuel, chanté librement. On ne chante jamais des vers en guidant les bœufs. L'aboio n'est pas un divertissement. C'est une vieille chose, très ancienne, respectée. On le chante dans la brousse pour l'orientation de celui qu'on cherche. On le chante assis sur la clôture du parc en regardant le troupeau rentrer. On le chante guidant le grand chef de la file, le soir ou le matin. On le fait pour le troupeau dans les champs et aussi pour le troupeau dans le parc, pour les vaches à lait, mais moins. Aboiar pour des vaches n'est pas le fait d'un vaqueiro qui se respecte et a le sens de l'honneur. (Dicionãrio de Folclore Brasileiro).

Nous renvoyons notre lecteur à la très longue note sur les différents aspects de l'aboio étudié par Luís da Câmara Cascudo dans son monumental Dicionário do Folclore Brasileiro ${ }^{13}$.

"Petit mot qui m'atteste je te lance tiaulé / dans le temps et les confins "; nous y retrouvons, comme par hasard, le climat du poème "inventaire de cayes". Lancer un mot tiaulé, inventaire à siffler sur la route : nous sommes devant deux poèmes à être chantés ou sifflés. Observons encore le titre du poème qui nous intéresse, "internonce ": non pas celui qui annonce (comme l'Ange de la Bonne nouvelle) mais celui qui interannonce, le médiateur, l'ouvreur des chemins, l'homme des carrefours, Eshu (celui qui vient sans qu'on l'appelle). Et Eshu (l'inommé, car son nom est tabou) représente la parole du narrateur, s'incarne dans un bestiaire d'eau et de mer, reliant le fond des abysses à l'espace de la plage et au haut des cieux, ouvrant la mort à la vie ; il est aussi plante fragile qui apporte le feu et brise les tombeaux. Eshu associé à la parole, créature " rebelle" du poète, prend d'assaut le temps-espace; son adjuvant est le sang de celui qui chante, "luciole parmi les lucioles". 
Notons encore que la conjonction Eshu-parole-sang s'enrichit de la charge connotative du pasteur qui par son chant dompte le troupeau. Il est fort possible que ce poème dans sa réception au Brésil actualise pour la plupart des lecteurs la conjonction Eshu/ parole tandis que sa réception chez des Africains restés en contact avec la tradition orale actualise surtout le savoir initiatique des Peuls, par exemple. Or, ce que le poème de Césaire réalise au niveau poétique c'est le syncrétisme de deux figures traditionnelles, celle du pasteur dominant son troupeau par son chant ou son sifflement (l'initié Peul, le vaqueiro profane), celle d'Eshu, entité d'origine africaine certes, mais réalité américaine enracinée à Cuba, en Haïti, au Brésil. L'une ou l'autre prégnance dépend du sens que le lecteur (Brésilien ou Africain) donne au mot tiaulé, à partir évidemment de son arrièreplan culturel. Il nous semble néanmoins que pour un Français de France, même après avoir consulté un dictionnaire savant quant aux mots tiauler, tiaulement, le passage restera non pas opaque mais sans chair. Pour mieux comprendre le phénomène et ses conséquences du point de vue pratique, nous renvoyons notre lecteur à un article au titre volontairement provocant, où nous soutenons l'intérêt d'une lecture où le lecteur assume son propre contexte culturel comme moyen d'appréhension anthropophage de l'œuvre étrangère ${ }^{14}$. C'est dans ce sens qu'une certaine prégnance de l'oralité chez le récepteur lui permet de mieux capter l'influence de l'oralité chez le producteur du texte.

Le poème no 23 qui, dans le dernier recueil césairien, précède l'énigme "ça, le creux ", s'intitule "mot-macumba ". Nous aurions sans doute intérêt à reprendre les textes classiques de Griaule sur Nommo dieu d'eau et Germaine Dieterlen (Essai sur la religion des Bambaras) mais ce serait encore une fois oublier le contexte beaucoup plus "américain" que proprement "africain" de moi, laminaire... La macumba n'est pas une réalité africaine, mais un culte du Nouveau Monde, un des produits du syncrétisme religieux au Brésil, exemple d'une religion populaire, en expansion, ouverte aux adaptations et transformant des éléments d'origines diverses.

En voici le texte :

Mot-macumba

le mot est père des saints

le mot est mère des saints

avec le mot couresse on peut traverser un fleuve peuplé de caïmans

il m'arrive de dessiner un mot sur le sol

avec un mot frais on peut traverser le désert d'une journée

il y a des mots bâton-de-nage pour écarter les squales

il y a des mots iguanes

il y a des mots subtils ce sont des mots phasmes

il y a des mots d'ombre avec des réveils en colère d'étincelles

il $y$ a des mots Shango

il m'arrive de nager de ruse sur le dos d'un mot dauphin.

\section{La traduction du poème :}

Palavra-macumba

a palavra é pai de santo

a palavra é mãe de santo

com a palavra cobra d'água pode-se atraverssar um rio cheio de jacarés às vezes risco uma palavra no chão

com uma palavra fresca pode-se atravessar o deserto de um dia

há palavras pau-de-afastar tubarão

há palavras iguanas

há palavras sutis são palavras fasmos 
há palavras de sombra com despertar de cólera e centelhas

há palavras Xangô

às vezes nado, astuto, sobre o dorso de uma palavra delfim.

Dans « mot-macumba », le mot a pour fonction de permettre de vivre et de survivre, adjuvant actif dans les dangers du fleuve, du désert et de la mer, contre les caïmans, la sécheresse et les squales. Père et mère des saints, il résume en lui tous les orishas. Plus : le mot n'est pas un, il est plusieurs. Il y en a de subtils (" mots phasmes ", littéralement : spectres), il y en a de violents (" mots d'ombre avec des réveils en colère d'étincelles »; "mots Sangho "). Dans ce poème qui a l'allure d'un ponto, l'intromission du narrateur (je) a lieu dans deux vers utilisant d'ailleurs la même formule, il $m$ 'arrive : "il m'arrive de dessiner un mot sur le sol "; " il m'arrive de nager de ruse sur le dos d'un mot dauphin".

La connaissance unique de la tradition chrétienne n'identifierait dans le geste de dessiner sur le sol un mot que le souvenir des premiers chrétiens se reconnaissant les uns les autres grâce au dessin d'un poisson. En effet, le mot grec ICHTUS est alors pris comme idéogramme, chacune des cinq lettres grecques étant regardée comme l'initiale d'autant de mots qui se traduisent : Jésus-Christ, Fils de Dieu, Sauveur (JESU KRISTUS THEOU VIOS SÔTER). Le souvenir se justifie sans doute, la macumba incorporant aussi des éléments chrétiens, du moins en surface. Mais, ici, le mot dessiné sur le sol est beaucoup plus proche du risco do ponto des orishas (ce dessin sur le sol rappelle le vévé haïtien), car au Brésil les chants rituels (pontos cantados) sont renforcés par des signes peints par terre avec la craie (pontos riscados), le dessin marquant le sol pour la descente d'un orisha (ou d'un loa) qui chevauche son cheval (c'est-à-dire, l'initié).

L'intromission du narrateur à la fin du poème ( il m'arrive de nager de ruse sur le dos d'un mot dauphin ") pourrait être interprétée grâce à la très riche iconographie d'Yemanja dans les Amériques noires et dont le lecteur trouvera de très beaux exemples dans le livre de Pierre Verger sur les orishas ${ }^{15}$. Césaire dans son poème "Lettre de Bahia ", écrit après son séjour au Brésil, paraît très sensible à l'iconographie de l'orisha des eaux fréquemment associé aux poissons et à la sirène ${ }^{16}$. Cependant, comme le dauphin apparaît dans sa poésie dès les premiers poèmes, le dernier vers (" il m'arrive de nager de ruse sur le dos d'un mot dauphin ") doit être lu aussi dans une intratextualité césairienne qui confirme, d'ailleurs, les grands axes de son symbolisme : le dauphin (fils du roi) lié à la régénérescence mais aussi le dauphin chevauché par un homme, symbole du héros sauveur.

Dans notre version du poème en portugais, après avoir beaucoup hésité, nous avons décidé de maintenir, du moins dans cet essai, comme traduction du mot français dauphin, son correspondant portugais delfim (à la fois, "fils du roi " et "espèce de cétacé », bien que dans cette acception le mot soit finalement peu employé ; on dirait plutôt golfinho ou boto). En écrivant " às vezes nado, astuto, sobre o dorso de uma palavra boto", nous infléchissons le texte du poème vers d'autres sens : avec boto nous abandonnons la signification " fils du roi » (et c'est une perte) mais le texte en portugais gagne en tonalité populaire et acquiert surtout la charge connotative et mythique des innombrables histoires sur le boto. Cette espèce de dauphin de l'Amazonie est appelé tour à tour boto, golfinho do Amazonas, boto branco, boto vermelho, piraia-guará, ou pira-iauara, peixe cachorro : l'inia geoffrensis (Blainville) est un cétacé fluvial de citation indispensable dans le folklore de tout le Nord brésilien. Le dictionnaire de Câmara Cascudo en fait 
un long inventaire, résumant les mythes, les tabous et les amulettes liés au boto. Nous n'en citons que le début :

Le boto séduit les filles dans toute la région des principaux affluents de l'Amazone, il est le père de tous les enfants de responsabilité inconnue. Les premières heures du soir, le boto devient un beau garçon, grand, blanc, fort, bon danseur et gros buveur : il apparaît dans les bals, séduit, parle, fréquente les réunions et va fidèlement à tous les rendez-vous féminins. Avant l'aube, il saute dans l'eau et reprend sa forme de boto.

Pour l'instant, nous proposons une version plus proche de l'original, bien que le titre même du poème fasse allusion à la macumba. Celle-ci justifierait le dérapage vers d'autres mythes, la macumba (de Rio) étant en général plus ouverte aux changements et aux syncrétismes avec d'autres mythes que le candomblé de Bahia, plus soucieux d'orthodoxie.

Avant d'aborder le poème de l'ensemble peut-être le plus prisé par la critique, "chanson de l'hippocampe", considérons encore quelques pièces dédiées à Wifredo Lam : "conversation avec Mantonica Wilson ", " connaître, dit-il ", "que l'on présente son cœur au soleil ", où le lecteur retrouve, surpris, la transposition de la tradition yoruba telle qu'elle s'est conservée par les Lucumi à Cuba et les Nagô au Brésil. D'ailleurs, dès le premier poème du tombeau, Wifredo Lam était présenté à la fois comme un frère et un initié : "liseur d'entrailles et de destins violets / récitant de macumbas / mon frère » $(M L ;$ p. 83).

Nous verrons que le premier des poèmes qui nous intéressent gravite autour d'Eshu, principe dynamique et principe de l'existence individualisée dans le système nagô. L'identification de l'artiste (créateur humain) avec Eshu est fort nette dans le deuxième poème du tombeau : "conversation avec Mantonica Wilson ». Mantonica Wilson est la marraine de Lam. Césaire cite en exergue de ses poèmes dédiés au peintre cubain, un passage de Lam lui-même :

Mantonica Wilson, ma marraine, avait le pouvoir de conjurer les éléments... Je l'ai visitée dans sa maison remplie d'idoles africaines. Elle m'a donné la protection de tous ces dieux : d'Yemanja, la déesse de la mer, de Shango, dieu de la guerre compagnon d'OgounFerraille, dieu du métal qui dorait chaque matin le soleil, toujours à côté d'Olorun, le dieu absolu de la création. (ML, p. 82)

Malgré ce qu'en dit Lam sur la protection des différents orishas Césaire, fidèle en cela à la tradition nagô, insiste sur le rôle d'Eshu à l'intérieur de ce système religieux.

\author{
Conversation \\ avec Mantonica Wilson \\ toi diseur \\ qu'y a-t-il à dire \\ qu'y a-t-il à dire \\ y pourvoit la tête de l'hippotrague \\ y pourvoit le chasse-mouche \\ toi diseur \\ qu'y a-t-il à dire \\ qu'y a-t-il à dire \\ la vie à transmettre \\ la force à repartir \\ et ce fleuve de chenilles
}




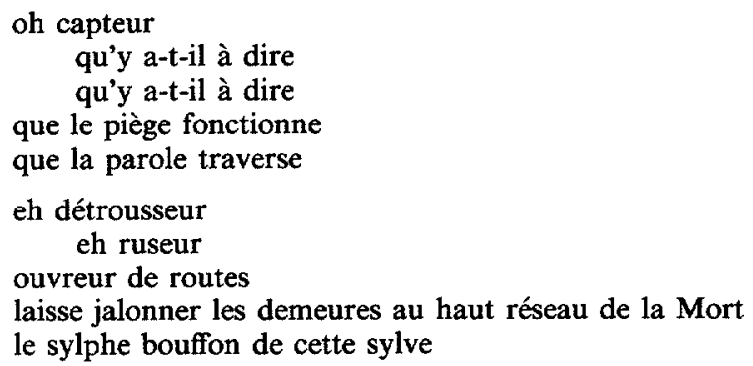

Nous proposons la version suivante de ce poème en portugais :

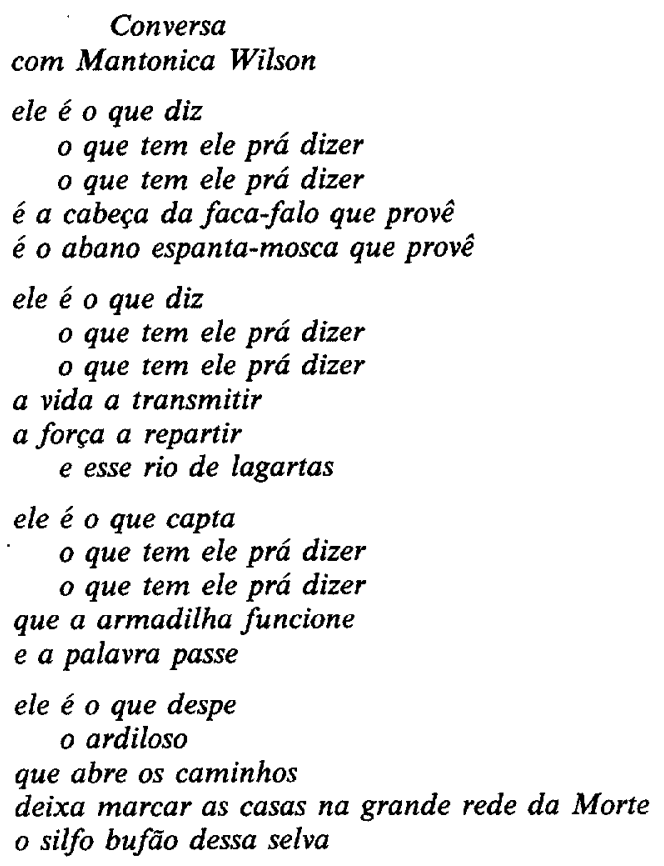

Des vocatifs (" toi diseur ", " oh capteur ", " eh détrousseur ", " eh ruseur ouvreur des routes", saluent celui dont le nom n'est pas cité, Eshu, mieux Legba. Car entre les Fon du Dahomey, Eshu-Elegbara a nom Legba. Ceux qui le reçoivent, les Legbasi, portent une jupe de paille teinte en violet et plusieurs colliers de cauris. Sous la jupe, les Legbasi portent un grand phallus en bois qu'ils lèvent avec des mimiques obscènes (c'est Eshu Elegbara qui fait irruption, sans être appelé, dans la fête organisée par Prospéro) ou encore le chasse-mouche qu'ils agitent sous le nez des assistants (Verger, Orishas, p. 79).

Tout caractérise Eshu Elegbara, même sa tête d'" hippotrague ". Le dieu africain porte en effet ses cheveux réunis dans une longue tresse sur le haut de la tête formant une sorte de "crête pour cacher la lame de couteau qu'il a sur le haut du crâne " (d'où notre traduction en portugais : "é a cabeça de faca-falo que provê »). Cela explique, comme l'indique Verger, ce qu'on dit d'Eshu dans un de ses orikis : Sonso abè kò lòri 
eèrù, c'est-à-dire, " le couteau sur la tête, c'est pour cela qu'il ne peut porter des fardeaux " (ibid., p. 78). Et Césaire le sait, car il récite dans un passage d'Une tempête :

Eshu n'est pas une tête à porter des fardeaux,

c'est un gaillard à la tête pointue. Quand il danse

il danse sans remuer les épaules.

Ah! Eshu est un luron joyeux! (Une tempête, III, 3, p. 70.)

Reste à résoudre le problème le plus intéressant : dans ce poème "conversation avec Mantonica Wilson ", qui parle à qui ? Pour le comprendre, essayons de cerner Eshu en tant qu'élément dynamique, non seulement de tous les êtres surnaturels, mais de tout ce qui existe. Dans ce sens, il est à la fois un et plusieurs. Nous citons un assez long passage de Juana Elbein dos Santos : elle y explique qu'Eshu est l'asè, c'est-à-dire la force qui propulse tout le système nagô, il est partout mais les êtres humains ne peuvent voir leur Eshu particulier. Dans ce cas, il faudrait sans doute comprendre que la marraine Mantonica Wilson s'adresse à l'Eshu de son filleul et que celui-ci parle à la marraine (qui " avait le pouvoir de conjurer les éléments », d'après Lam lui-même). Voici le texte de Juana Elbein dos Santos :

... comme Olorun, l'entité suprême protomatière de l'univers, Eshu ne peut être isolé ou classé dans une catégorie. C'est un principe et, comme l'asè qu'il représente et porte, il participe forcément à tout. Principe dynamique et d'expansion de tout ce qui existe, sans lui tous les éléments du système et son devenir resteraient immobiles, la vie ne se développerait point. Selon les mots d'Ifa, " chacun a son propre Eshu et son propre Olorun dans son corps " ou "chaque être humain a son Eshu individuel, chaque ville, chaque maison (lignage), chaque entité, chaque chose et chaque être a son Eshu ", et encore, "si quelqu'un n'avait pas son Eshu dans son corps, il ne pourrait exister, ne saurait pas qu'il était en vie, parce qu'il faut que chacun ait son Eshu individuel ». Ainsi comme Olorun représente le principe de l'existence générique, Eshu est le principe de l'existence différenciée en conséquence de sa fonction d'élément dynamique qui le fait mouvoir, développer, mobiliser, grandir, transformer, communiquer (...) Seuls les êtres humains ne peuvent voir leur Eshu particulier (...) Nous voyons, ainsi, à partir des textes ci-dessus, largement confirmés par la pratique liturgique, que la fonction d'Eshu consiste à apporter une solution, résoudre tous les "travaux ", trouver les chemins appropriés, les ouvrir et les fermer et, surtout, fournir son aide et pouvoir afin de mobiliser et développer non seulement l'existence de chaque individu mais aussi les tâches spécifiques attribuées et déléguées à chacune des entités surnaturelles.

(Os nagô e a morte, Vozes, 1976, pp. 130-132.)

Le poème " connaître, dit-il ", diffère des précédents, car il invoque et salue non pas une divinité, mais plusieurs, liées à la connaissance et à l'activité sexuelle. Par ordre d'entrée probable : Ogoun, Osain et le couple Shango-Oya Yansan.

connaître, dit-il

eh connaisseur du connaître

par le couteau du savoir et le bec de l'oiseau

eh dégaineur

par le couteau du sexe et l'oiseau calao

eh disperseur de voiles

ici

ici la croupe des femmes et le pis de la chèvre

ici

ici

par tout œil écorcheur de crépuscules 
l'orteil qui insiste

comme aux pistes de la nuit

l'ardent sabot du cheval-vent

En voici la traduction :

conhecedor, diz ele

ei conhecedor do conhecer

pela faca do saber e o bico de pássaro

ei desembainhador

pela faca do sexo e o pássaro calao

ei levantador de véus

aqui a anca das mulheres e a teta da cabra

aqui

aqui

aqui

por todo olho descarnador de crepúsculos

$o$ artelho que insiste

como nas pistas da noite

o casco ardente do cavalo-vento

Les vocatifs à intonation populaire (" eh connaisseur du connaître ", " eh dégaineur ", "eh disperseur de voiles ") précédés du $e h$ intime et populaire, et non pas du $\hat{o}$ classique et noble, saluent les orishas manifestés dans leurs chevaux qui dansent, en tant que révélateurs de ce qui est fermé, voilé ou caché : connaissance et sexe sont étroitement liés.

Le " couteau du savoir " se rattache à Ogoun dont le nom est toujours cité à l'occasion des sacrifices aux différents orishas, car il est le seigneur du couteau. Dans le candomblé, il est le premier à être salué après Eshu : dans les cérémonies du Nouveau Monde, il défile martialement, ouvrant la voie aux autres orishas, quand ils font leur entrée, les jours de fête, dans le barracão (cf. Verger, Orixás, p. 94).

Le « bec de l'oiseau » renvoie à Òsanyìn. Selon Verger, une histoire d'Ifa nous appprend que l'oiseau est la représentation du pouvoir d'Osain, l'orisha des plantes médicinales et liturgiques, dont le symbole est la verge en fer surmontée d'un oiseau en fer forgé entouré de six pointes en éventail. Le rapport Osain/oiseau est fort connu : l'oiseau " est son messager qui va partout, retourne et se perche sur la tête d'Osain pour lui faire son rapport. Ce symbolisme de l'oiseau est bien connu des sorcières, celles qu'on appelle souvent Eleye, maîtresses de l'oiseau-pouvoir» (ibid., p. 122). D'autre part, Osain se lie encore à Orounmila, le maître de la divination.

Dans le poème, le " couteau du savoir " isomorphe du " bec de l'oiseau » qui perce l'avenir, s'articule encore avec le couteau du sexe qui ouvre les corps (" la croupe des femmes ", "le pis de la chèvre "). Glissant, dans son Discours antillais, rappelle l'éventail des expressions par quoi la langue créole permet à un homme de se vanter d'avoir fait l'amour avec une femme : coupé famm'la, batt famm'la, raché famm'la, littéralement " couper la femme ", "battre la femme", " tailler la femme " (DA, p. 229). Dans le poème de Césaire, l'œil lui-même "écorche les crépuscules ", c'est-à-dire lui enlève son écorce, le dépouille de sa peau. Notons cependant que cette action n'est pas mutilante, elle est essentiellement ouvrante et fécondante. Elle révèle et dévoile.

Le rappel au sexe pourrait s'articuler soit avec la fécondité concrète (chez l'homme et la femme), soit avec la fécondité mystique (Osain), soit avec la fécondité primordiale (Oshala), mais la présence à la fois de la femme ( la croupe des femmes"), d'un animal du type de la chèvre (bélier) et le cheval-vent (l'une des manifestations 
d'Oya-Yansan, l'une des trois femmes de Shango) orientent notre lecture vers Shango, roi-guerrier monté sur le cheval-vent. C'est d'ailleurs sous cet aspect qu'il apparaît dans Une tempête grâce aux chants de Caliban :

Shango est l'ameuteur de pluies

Bien enveloppé il passe dans son manteau de feu.

Des pavés du ciel le sabot de son cheval

tire des éclairs de feu.

Shango est un grand cavalier

Shango Shango ho!

(Une tempête, III, 4, p. 75.)

Et encore :

Shango marche avec force

à travers le ciel, son promenoir !

Shango est un secoueur de feu

chacun de ses pas ébranle le ciel

ébranle la terre

Shango Shango ho

(Une tempête, III, 5, p. 89.)

Dans le poème "que l'on présente son cœur au soleil » (ML, p. 92), lui aussi dédié à Wifredo Lam, nous retrouvons en même temps le geste des prêtres aztèques qui présentaient le cœur de leurs victimes au soleil et la grande Bête du folklore antillais. Voici le texte du poème :

Que l'on présente son cour au soleil

la Bête a dû céder sur le sentier de ton dernier défi

Bête aux abois

Mort traquée par la mort

de son masque déchu elle s'arc-boute à son mufle

solaire

l'œuf la suit à la piste

l'aile du tout-à-coup jaillit

la victoire est d'offrir à la gourde des germes

le sexe frais du temps

sur l'aube d'une main mendiante de fantômes

En voici la traduction :

Apresentem seu coração
ao sol

a Besta teve que ceder no caminho do teu último desafio

Besta em agonia

Morte encurralada pela morte

a máscara caída ela retesa o focinho

solar

o ovo segue sua pista

a asa do de repente jorra 
vencer é oferecer na cabaça dos germes

o sexo fresco do tempo

sobre a aurora da mão mendicante de fantasmas

Wifredo Lam, tantôt salué comme Eshu, est maintenant le nouveau Ti-Jean l'Horizon, vainqueur de la Bête. Le premier vers suggère l'affrontement entre le héros et la Bête maléfique. La Mort identifiée à la Bête est vaincue par le héros qui meurt : nous y retrouvons un schéma obsessif chez Césaire, depuis le Cahier et qui traverse toute son cuvre poétique et théâtrale, l'inversion de l'inversion : le héros vaincu, par sa mort, vainc le Mal, la Mort, la Bête. Le peintre Wifredo Lam rejoint ici les figures de Toussaint (Cahier), Lumumba (Une saison au Congo), le Rebelle (Et les chiens...), etc.

La forme aveuglante et ronde du soleil est identifiée au moment même de la mort du héros, à l'œuf cosmique, compris en tant que germe à partir duquel se développe la naissance du monde, idée commune, comme l'indique Chevalier dans son Dictionnaire de symboles, à de nombreux peuples du monde entier. Mais ce qui nous intéresse ici c'est de montrer qu'Eshu, auquel s'identifie Lam si souvent dans les poèmes de moi, laminaire..., s'articule dans le système nagô à l'œuf, à l'utérus, à l'itération du sperme et de l'ovule. Juana Elbein dos Santos écrit :

Eshu a des rapports avec l'activité sexuelle (...) et avec l'itération de la semence et de l'ovule. Il est encore fondamentalement lié au placenta fécondé. Tandis que l'utérus et la semence représentent les matières-masses d'origine, les ancêtres mythiques féminins et masculins, le placenta transmet le principe de vie individuelle (...) le placenta est le double de l'individu nouveau, il se développe avec le photus et, séparé du corps maternel, deviendra un symbole d'Eshu. (Ibid., p. 212.)

Lorsque nous avons analysé le poème-devinette " ça, le creux ", nous avons choisi justement comme exemple d'énigme religieuse africaine, celle d'Orunmila et l'œuf : "Elles disent, lancer ; Orunmila dit attraper et ceci sept fois ". C'était pour nous un moyen de restreindre le nombre des citations, l'œuf étant un symbole fort riche dans la traditon nagô.

La deuxième strophe du poème « que l'on présente son cœur au soleil » décrivant la mort de la Mort correspond en fait à une fin de monde, une eschatologie, tandis que les strophes suivantes qui ont comme centre l'œuf, l'aile, la conjonction sexuelle suggèrent l'émergence d'un nouveau monde, une cosmogonie, avec le surgissement à la lumière de nouveaux êtres formés à partir de "fantômes " (c'est-à-dire, des morts). Nous y retrouvons une caractéristique essentielle de la cosmogonie césairienne, fête d'Eros ${ }^{17}$. Par là s'explique l'image finale de l'" aube » où les mains mendiantes des morts aspirent à se réincarner, à réapparaître sur terre dans le temps des hommes grâce à l'activité sexuelle.

"La chanson de l'hippocampe", dixième poème de moi, laminaire..., réunit à la fois le ton oral, le thème du cheval et la lutte d'un être apparemment fragile contre les forces maléfiques. L'hippocampe surgit peut-être du souvenir d'un passage du Bateau ivre, "planche folle, escorté des hippocampes noirs", mais dans la série de poèmes du recueil celui-ci s'articule directement avec le poème précédent ( ${ }^{\circ} 9$, " mangrove ") qui s'achevait sur le désir qu'un cheval surgisse de l'eau immobile de la mangrove. 
Rappelons-nous la fraîcheur de la chanson :

Chanson de l'hippocampe

petit cheval hors du temps enfui

bravant les lès du vent et la vague et le sable turbulent

petit cheval

dos cambré que salpêtre le vent

tête basse vers le cri des juments

petit cheval sans nageoire

sans mémoire

débris de fin de course et sédition de continents

fier petit cheval têtu d'amours supputées

mal arrachées au sifflement des mares

un jour rétif

nous t'enfourcherons

et tu galoperas petit cheval

sans peur

vrai dans le vent le sel et le varech

Nous proposons la traduction :

Canção do hipocampo

cavalinho além-tempo foragido

arrostando as dobras do vento e a onda e a areia turbulenta

cavalinho

dorso arqueado que o vento cobre de salitre

cabeça baixa na direção do guincho das fêmeas

cavalinho sem barbatana

sem memória

restos de fim de corrida e sedição de continentes

altivo cavalinho teimoso de amores suputados

mal arrancados do assobio das poças

um dia insubmisso

nós te cavalgaremos!

e galoparás cavalinho

sem medo

de verdade ao vento no sal no sargaço

Dans les contes créoles, il arrive que de petits animaux faibles luttent contre les puissants. Ils en sont écrasés. Le modèle achevé en est Conte Colibri. À Colibri, Césaire ajoute une sorte de Conte Hippocampe où le petit cheval, vainqueur de l'adversité, sera un jour enfourché et entrera dans le futur.

Le cheval si important dans l'imaginaire césairien, galopant comme le sang dans les veines, surgi des entrailles de la terre ou des abysses de la mer, destructeur et triomphateur, lié à l'impétuosité du désir, subit ici un processus de miniaturisation. Le syntagme "petit cheval "se réitère cinq fois dans ce court poème-chanson et devient la monture de la révolte et de la liberté.

Ce petit cheval à la fois enfui/enfoui hors du temps vient de très loin, d'époques révolues. Son attitude est celle de la résistance et du défi. Il vit sous l'influx du désir dans sa double carence : "sans nageoire " (sans aide donc pour nager), "sans mémoire » (ce qui revient à dire sans conscience de son histoire). Cette forme primitive, gracile et fragile, vit entre deux temps : entre la fin d'un monde (" débris de fin de course et sédition 
de continents ») et la recréation du monde. La suggestion de la création du monde vient du « sifflement des mares " où se profile le contour du serpent primordial dans l'utérus des eaux féminines. Un jour qui tarde à apparaître (" jour rétif »), le nous collectif prendra le petit cheval comme sa monture et galopera «sans peur vrai dans le vent le sel le varech".

$\mathrm{Au}$ terme de ce bref inventaire, dégageons rapidement quelques conclusions provisoires. Notre but ici était d'envisager le dernier livre de poèmes de Césaire d'un point de vue assez simple : attirer l'attention du lecteur sur la tonalité " américaine " du recueil sous l'influence de l'oralité. En effet, Césaire l'Africain s'" américanise" le soir de sa vie : les hommes/créateurs qu'il chante (Fanon, Damas, Asturias, Lam) sont tous des Antillais ; la plupart des divinités qu"il invoque sortent des cultes syncrétiques du Nouveau Monde et non pas de l'Afrique, mère ancestrale; ses héros, populaires, sont ceux des contes créoles (l'oiseau magique, Ti-Jean l'Horizon); le paysage qu'il décrit (mangrove, volcan, mornes, cayes, récifs de corail) appartient à l'Amérique tropicale en général, aux Caraibes en particulier, avec sa faune (piranha, couresse, pacarana, malfini, ravet) et sa flore (mangle, drago, mancenillier) ; c'est enfin dans moi, laminaire... où l'on trouve les poèmes sans doute les plus " oraux " de Césaire, non pas uniquement grâce au travail souterrain de la syntaxe créole, mais encore grâce à l'emploi de formes traditionnelles (conte, comptine, devinette, oriki, etc.). Le livre achevé, en en relisant les paroles liminaires, les lecteurs voient que, comme l'initié (qu'il soit Haïtien, Cubain ou Brésilien), le poète fait son offrande aux quatre directions de l'espace ${ }^{18}$ (l'orient et l'occident, le nord et le sud : le futur, le destin personnel, le géniteur divin, l'Eshu individuel et les ancêtres; les éléments masculins et les éléments féminins) au carrefour desquelles se place « l'inégale lutte de la vie et de la mort, de la ferveur et de la lucidité, fût-ce celle du désespoir et de la retombée, la force aussi toujours de regarder demain » (ML, p. 9). Mais se retourner vers les quatre directions cardinales peut avoir aussi un autre sens : ce geste se répète à plusieurs moments des rites, en particulier lorsque le babalaô ( le père du secret 》) jette les 16 cauris pour savoir ce que réserve le destin à celui qui le consulte. Et dans le jeu des cauris c'est Eshu qui répond (cf. Verger, ibid., p. 127).

Les premières versions du Cahier d'un retour au pays natal et les Pur-sang étaient, on le sait maintenant ${ }^{19}$, marquées par la lecture de l'œuvre de Léo Frobenius sur l'Afrique. Les derniers poèmes de Césaire portent la trace, indéniable, d'un contact direct avec la terre et les cultes américains (le candomblé, la macumba au Brésil ; le Lucumi cubain; le voudoun haïtien). À d'autres de suivre la piste ${ }^{20}$.

Notes

1. Pestre de Almeida, Lilian (1982) : la Poétique de Césaire, inédit.

2. Id. et aussi $O$ Teatro Negro de Aimé Césaire (1978) : Niterói, UFF.

3. Hale, Thomas A. (1984) : «Littérature orale : le discours comme arme de combat chez Aimé Césaire ", in Soleil éclaté, Tübingen, Gunter Narr Verlag, pp. 173-186.

4. Rio, Difel (1976).

5. Laroche, Maximilien (1981) : « La diglossie dans Gouverneurs de la rosée : termes de couleur et conflit de langues ", in la Littérature haîtienne; identité-langue-réalité, Ottawa, Leméac.

6. Bernabé, Jean (1982) : "Le travail de l'écriture chez Simone Schwartz-Bart. Contribution à l'étude de la diglossie littéraire créole-français ", in Présence africaine, $\mathbf{n}^{\mathrm{O}} 121 / 122$, 1 $1^{\mathrm{er}}$ et $2^{\mathrm{e}}$ trimestres, pp. 166-179.

7. Inutile de dire que la norme brésilienne ne coïncide pas toujours avec la norme portugaise. Au Brésil, tout un chacun sait, de science infuse et certaine, qu'il y a plusieurs portugais. Pour n'importe quel Brésilien celui qui a un drôle d'accent et parfois une drôle de syntaxe est toujours le Portugais, jamais nous. On n'éprouve donc pas le besoin de dire qu'on parle le "brésilien ".

8. Os nagô e a morte (1976) : Petrópolis, Vozes.

9. Gouverneurs de la rosée (1946) : Paris, les Éditeurs réunis.

10. Théoda (1958) : Albeuve (Suisse), Éd. Castella. 
11. Une amie anthropologue, Elena Andrei, me suggère de traduire le passage " encore un mauvais coup d'Eshu » par " mais uma surpresa de Exu Tiriri". Ses arguments : a) on maintiendrait ainsi et le nom d'Eshu et le jeu sur les $i$; b) Tiriri est le nom d'un Eshu (il y en a plusieurs) d'Ogoun et d'Oshosi ; son signe : il a les jambes tordues et se lie à la vengeance, à la haine. Qu'elle en soit ici remerciée : la suggestion me paraît bonne mais seuls les initiés connaissent ce nom Tiriri ; par contre, le nom de Pomba Gira fait partie du vocabulaire courant. C'est la seule raison qui nous fait encore hésiter. Traduire est toute une aventure.

12. Le dictionnaire espagnol de la Real Academia registre : Drago, "arbre de la famille des liliacées, originaire des îles Canaries, dont on obtient, à partir d'incisions sur son tronc, la résine appelée « sangre de drago" employée en médecine".

13. Câmara Cascudo, Luís (1972) : Dicionário do Folclore Brasileiro, $3^{a}$ edição, MEC, Instituto Nacional do Livro.

14. Pestre de Almeida, Lilian (1984) : "Défense et illustration de l'anthropophagie ", in Césaire 70, Paris, Silex Éditions, pp. 123-140.

15. Verger, Pierre Fatumbi (1981) : Orixás, Deuses iorubás na África e no Novo Mundo, Salvador, Corrupio.

16. Cf. Verger, ibid., pp. 202-203.

17. Pestre de Almeida, Lilian (1984) : "La cosmogonie césairienne, fête d'Eros" (Pour une lecture de l'imaginaire césairien), in Soleil éclaté, Tübingen, Gunter Narr Verlag, pp. 333-350.

18. Pour les quatre points de l'univers et leurs correspondances dans le système nagô voir Juana Elbein dos Santos, op. cit., p. 183. Dans de nombreux passages de Gouverneurs de la rosée, les personnages se tournent rituellement vers les quatre points cardinaux. Voir, entre autres le passage :

Avant de semer le maïs, au lever du matin, devant l'ceil rouge et vigilant du soleil, elle (Délira) avait dit au Seigneur Jésus-Christ, tournée vers le levant, aux Anges de Guinée, tournée vers le sud, aux Morts, tournée vers le couchant, aux Saints, tournée vers le nord, elle leur avait dit, jetant les grains aux quatre directions sacrées : Jésus-Christ, les Anges, les Morts, les Saints : voici le maïs que je vous donne, donnez-moi en retour le courage de travailler et la satisfaction de récolter. (Gouverneurs de la rosée, p. 62.)

19. Steins, Martin (1984) : "Die Geburt der Negritude aus dem Geist des Krieges " Aimé Césaire Gedicht les Pur-sang und Leo Frobenius, in Neohelicon, XI 2, pp. 83-125.

20. Après la découverte d'Haïti en 1947, Césaire n'est pas revenu dans l'île où " ia négritude se mit debout ", ne voulant probablement pas accorder, par sa présence, une caution au régime Duvalier. Il nous semble que dans moi, laminaire... prédominent des éléments d'origine soit "brésilienne", soit " cubaine ", plus qu'« haïtienne ". Mais l'impression, à approfondir, devrait être confirmée ou contestée par des recherches plus poussées. 